MINIREVIEW

\title{
Molecular elements of low-oxygen signaling in plants
} Francesco Licausi

Scuola Superiore Sant'Anna, 56124 Pisa, Italy, f.licausi@sssup.it

Received 9 September 2012; revised 28 October 2012

doi:10.1111/ppl.12011

\begin{abstract}
Oxygen and its limitation are emerging as a crucial factor in plant fitness, growth and development. Recent studies revealed the mechanisms by which oxygen is perceived by plant cells. This sensory system partly relies on an oxygen-mediated branch of the $\mathrm{N}$-end rule pathway for protein degradation acting on a specific clade of ethylene responsive transcription factors (ERF-VII). A complementary regulative step is provided by aerobic sequestration of an ERF-VII protein at the plasma membrane and its timely release when hypoxia occurs. Complete absence of oxygen triggers the transient accumulation of reactive hydrogen peroxide and induces an additional set of reactive oxygen species-related genes involved in both signaling and attenuation of oxidative stress. Moreover, temporary hypoxic environments that are built up as consequence of dense cell packing have been demonstrated to trigger cell-fate determination in maize anthers. Similarly, limited oxygen delivery in bulky fruit or tuber tissues growing in aerobic conditions were shown to stimulate anaerobic-like responses. These advances in low-oxygen signaling and its effect on cell development highlight the importance of taking hypoxia into account in agronomical practices as well as in breeding programs.
\end{abstract}

\section{Introduction}

Despite being able to produce oxygen from photosynthesis, plants also require this gas as final acceptor of electrons in the respiratory chain to produce energy. Oxygen can become limiting as a result of reduced diffusion because of constraints imposed by growth and development or when its concentration in the surrounding environment decreases below the consumption rate (Bailey-Serres et al. 2012). Being sessile organisms, plants cannot escape sudden water logging or deep flooding, conditions that strongly limit oxygen diffusion and thus its availability for the submerged tissues. Therefore, plants need to rapidly activate a metabolic reset to avoid complete blockage of energy production
(Bailey-Serres and Voesenek 2008). Because anaerobic metabolism is not as efficient as the aerobic one, developmental and growth programs need to be adjusted to cope with the reduction in ATP availability. A core of about 50 genes that respond ubiquitously to oxygen deficiency has been identified by microarray analyses in several plant species (Mustroph et al. 2010). This includes genes involved in fermentative and alanine metabolism, scavenging and detoxification of reactive oxygen and nitrogen species as well as a big number of proteins of unknown function (Roberts et al. 1989, Igamberdiev and Hill 2004, Mustroph et al. 2010, Rocha et al. 2010).

Until 2011, the major achievements in the research field of plant anaerobiosis dealt with species-specific mechanism able to provide tolerance or escape

Abbreviations - ACBP, acyl-CoA-binding protein; ACO, ACC oxidase; ACS, ACC synthase; ADH, alcohol dehydrogenase; $A T E$, arginyl transferase; ERF, ethylene response factor; GFP, green fluorescent protein; HB1, non-symbiotic hemoglobin 1; HRE, hypoxia responsive ERF; HSF, heat shock factor; HSP, heat shock protein; MAC1, multiple archesporial cells 1; MAP, mitogen-activated kinase; MC, methionine-cysteine; NERP, N-end rule pathway; NO, nitric oxide; RAP2, related to APETALA2; RBO, NADPH/respiratory burst oxidase protein; ROP, rho of plant; ROP2, RHO-like small G protein of plant; PDC, pyruvate decarboxilase; ROS, reactive oxygen species; SUB1, submergence1; TF, transcription factor. 
properties in rice (Xu et al. 2006, Hattori et al. 2009, Lee et al. 2009), rather than concerning cross-species conserved mechanisms of hypoxia perception and signal transduction. Pioneering discoveries in this second direction arose from a combination of transcriptomic approaches carried out in Arabidopsis thaliana, rice (Oryza sativa) and poplar (Populus trichocarpa) (Mustroph et al. 2010). As result, a series of reports were produced that dealt with the identification and characterization of transcription factors (TFs) that able to enhance the expression of subsets of anaerobic genes, although not strictly required for their induction upon hypoxia (for a review see Licausi 2011). Among them, ethylene response factors (ERFs) caught the attention of several research groups (Licausi et al. 2010, Peña-Castro et al. 2011). In fact, genes encoding group-VII ERFs recurred as upregulated in the anaerobic transcriptome of several plant species (Nakano et al. 2006, Mustroph et al. 2010). This interest was further enhanced by the identification of ERF proteins as key players in the different adaptation to flash flooding and long-lasting submergence in different rice varieties (Xu et al. 2006, Hattori et al. 2009).

\section{The $\mathbf{N}$-end rule pathway and ERF-VII are components of the oxygen signaling pathway}

The existence of a direct mechanism of oxygen perception and hypoxic signal transduction in plants has been highly debated. Because an ortholog of the animal oxygen sensor PHD1-HIF or the fungal SRN1 was not present in plants, mechanisms of indirect oxygen perception were proposed (Licausi and Perata 2009, Mustroph et al. 2010). According to these hypotheses, the alteration of metabolic parameters associated with oxygen deficiency would have triggered the molecular response to anaerobiosis. However in 2011, two groups independently reported the identification of an oxygen sensing mechanism able to trigger the molecular response to hypoxia and tuned itself by the oxygen availability within a cell (Gibbs et al. 2011, Licausi et al. 2011b). Both groups identified the conserved $\mathrm{N}$-terminal motif (MCGGAI/L) of group VII ERFs as an ideal substrate for the $\mathrm{N}$-end rule pathway (NERP) for protein degradation, a ubiquitous mechanism in eukaryotes and prokaryotes that dictates the stability of polypeptides depending on the exposed amino-terminal residue (Fig. 1) (Gibbs et al. 2011, Licausi et al. 2011b). The cysteine residue in second position, followed by neutral and non-encumbering glycine provides the perfect environment for methionine removal by aminopeptidases (Liao et al. 2004). This leaves a $\mathrm{N}$-terminal cysteine residue exposed to oxidizing agents such as oxygen and nitric oxide $(\mathrm{NO})$ that lead to the formation of cysteine sulfinic or sulfonic acid (Kwon et al. 2002). Oxidized cysteine subsequently acts as substrate for arginine-tRNA protein transferases (ATEs) for the addition of an arginyl residue (Arg) at the N-terminus via peptide bond. In turn, Arg signals a class of ubiquitin ligases (PRTs) to polyubiquitinate surrounding Lys residues thereby promoting the degradation of the polypeptide by the $26 S$ proteasome (Bailey-Serres et al. 2012). The observation that $\mathrm{NO}$ synthesis is increased under oxygen limitations disfavors its involvement in mediating aerobic degradation of ERF-VII proteins, although it still plays a role as modulator of the anaerobic response (Stöhr and Stremlau 2006). Oxygen is therefore more likely to be the direct trigger of ERF-VII proteolysis. Interestingly, non-symbiotic hemoglobin 1 (HB1), a NO-scavenging enzyme, is a direct target of RAP2.12 (Dordas et al. 2003, Licausi et al. 2011b). It is tempting to speculate that this protein may reduce $\mathrm{NO}$ levels under anoxia to antagonize the oxidation of the terminal Cys in ERF-VII TFs.

Gibbs et al. (2011) indeed identified the hypoxia inducible ERFs HRE1 and HRE2 as substrates for the $\mathrm{N}$-end rule in vitro, but not mutant versions of them where the penultimate Cys was substituted with alanine. One year earlier, we attempted a first physiological characterization of HRE1 and HRE2 by overexpressing both in Arabidopsis (Licausi et al. 2010). However, as these transgenic plants expressed the wild-type version of these genes, the NERP prevented accumulation of the respective protein, thus minimizing their impact on the Arabidopsis transcriptome. Nevertheless, we observed that under hypoxic conditions the induction of most of the anaerobic genes was enhanced as compared to wild-type plants (Licausi et al. 2010). A very similar observation was reported by Hinz et al. (2010), where the ERF-VII RAP2.2 was overexpressed instead. Interestingly, effective accumulation of ERF-VII protein in planta was reported by Papdi et al. (2008) where the ERF RAP2.12 was placed under control of an estradiol inducible promoter. In this case, significant upregulation of the $A D H$ gene was detected, suggesting that a sudden and massive increase in the synthesis of RAP2.12 was able to surpass its degradation rate via the NERP. This was also observed when we tried to express an HAtagged version of RAP2.12 in A. thaliana; in this case a whole transcriptome analyses revealed that the whole set of the core response to hypoxia was upregulated in these transgenic plants already in normoxic conditions (Licausi et al. 2011b). This series of experimental results highlighted the importance of ERF-VII in the induction of the anaerobic response but has left unanswered the question about its real triggers. It seems unlikely that HRE1 and HRE2 can be the first actors in the signaling cascade during hypoxia. First of all, they are induced by 


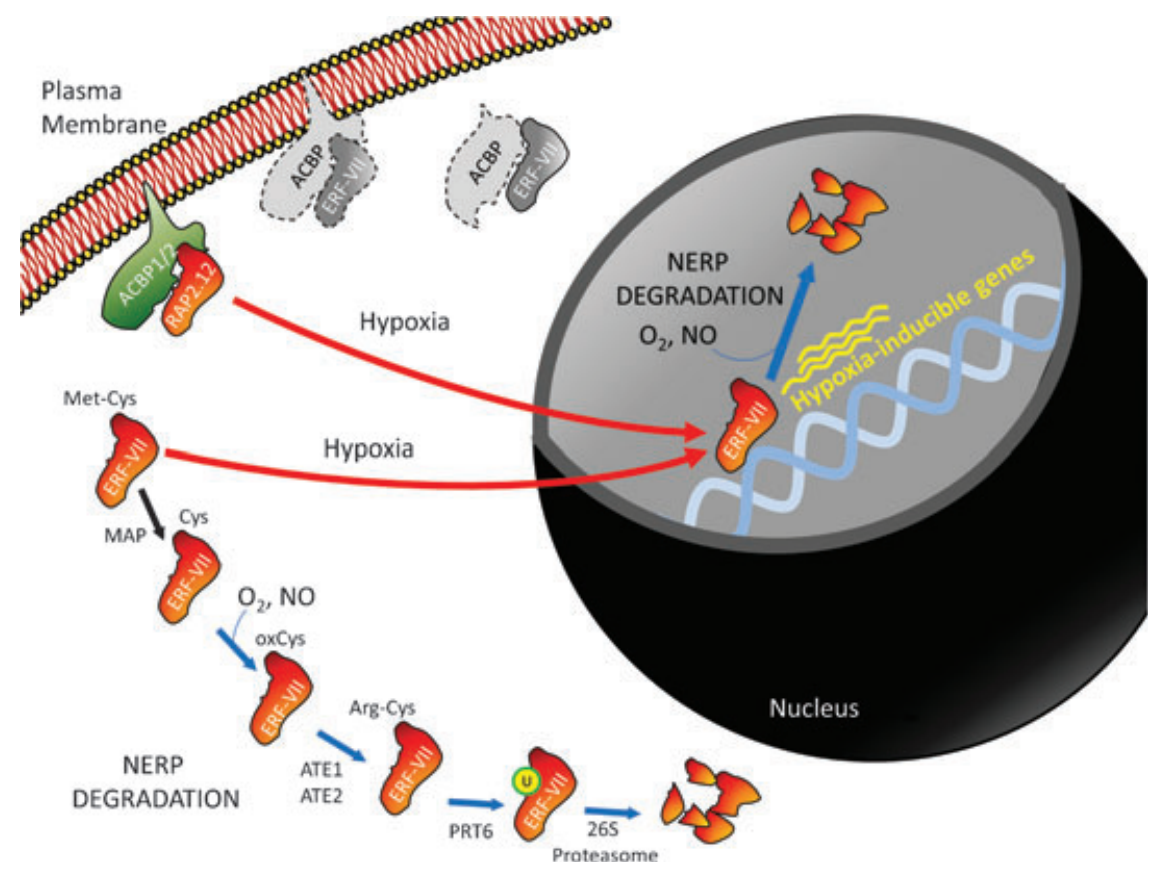

Fig. 1. Schematic representation of oxygen signaling in plants. RAP2.12 is bound to the membrane localized acyl-CoA binding protein 1 and 2 (ACBP1 and ACBP2). Potentially, other ERF-VII proteins (dark gray) interact with membrane and cytosol located ACBPs. N-terminal methionine is removed from ERF-VII proteins by methionine aminopeptidase (MAP) leaving a cysteine residue exposed. Under aerobic conditions (blue arrows), ERF-VII proteins are degraded following the NERP. In detail, the N-terminal cysteine is oxidized in the presence of oxygen or nitric oxide (oxCys), thereby providing a substrate for the addition of arginine via arginine-tRNA protein (ATE1 and ATE2). N-terminal arginine promotes polyubiquitination (indicated by $U$ ) and subsequent degradation via the $26 \mathrm{~S}$ proteasome. Upon hypoxia (red arrows), reduction of oxygen levels prevent the degradation of ERF-VII, which then move into the nucleus where they activate the response to anaerobiosis. Upon reoxygenation, ERF-VII proteins are rapidly degraded via the NERP.

hypoxia themselves and expressed at a negligible level under non-stress conditions. Moreover, HRE1 is mostly induced in non-photosynthetic tissues, while HRE2 appears to be ubiquitously upregulated. Anyway, a double knock-out mutant for both genes was perfectly able to induce the anaerobic response to levels comparable to those of wild-type plants, ruling these two genes out as main actors in triggering the anaerobic response. Instead, their importance in Arabidopsis seems to be associated to the maintenance of the anaerobic response, as the respective double mutant was not able to transcribe the anaerobic genes after few hours from the onset of anoxia, while wild-type plants could (Licausi et al. 2010).

Instead, transgenic plants in which the expression of both RAP2.2 and RAP2.12 was reduced via an artificial microRNA showed a lower induction of the anaerobic genes (Licausi et al. 2011b). Interestingly, also the expression of HRE1 and HRE2 resulted hampered in these silenced plants, suggesting that the two inducible ERFs are induced by the RAP genes (Licausi et al. 2011b). It certainly would be interesting to observe the ability of a true double knock-out for both RAP2.2 and RAP2.12 to activate the anaerobic response. A fifth member of the
ERF-VII group exists in the Arabidopsis genome: RAP2.3. The corresponding gene is transcribed at very high levels and thus was one of the first ERF to be described (Büttner and Singh 1997). It has been demonstrated to be required to alleviate oxidative stress in plants and so far no report linked it to the anaerobic response, albeit its $\mathrm{N}$-terminal sequence makes it also a potential NERP substrate.

The rapid induction of anaerobic genes in Arabidopsis already after 30 min hypoxia suggested that a signal should be ready to be released in the nucleus (Licausi et al. 2011c). Indeed, low amounts of RAP2.12 were observed in the plasma membrane of aerobic cells by using a RAP2.12:Green Fluorescent Protein (GFP) reporter fusion (Licausi et al. 2011b).The GFP signal was then accumulating in the nucleus when the oxygen concentration was decreased artificially. RAP2.12 does not contain any transmembrane domain that would justify its localization at the plasma membrane site. However, Li et al. (2008) demonstrated that ERF-VII protein can interact with membrane-associated acylCoA-binding protein (ACBPs). In fact, the interaction between RAP2.12 and ACBP1 and 2 was confirmed by yeast two-hybrid and bimolecular fluorescence 
complementation (Licausi et al. 2011b). It remains to be defined whether this interaction is disrupted upon low oxygen or the ACBP-RAP2.12 complex moves as a whole into the nucleus. The observation by $\mathrm{Li}$ et al. (2008) that a GFP-tagged version of ACBP2 accumulated at the periphery of the nucleus disfavors this hypothesis. It is also not yet clarified whether RAP2.12 requires ACBP1 and ACBP2 to be associated with the membrane, or their interaction occurs after the localization of RAP2.12 at the plasma membrane, maybe through association with other membrane bound proteins.

\section{Hormonal regulation of ERF-VII TFs}

Because low-oxygen stress is often occurring as consequence of flooding or submergence, a condition in which gases diffuse 10000-fold slower (Armstrong, 1979), researchers focused their attention to the involvement of ethylene in the low-oxygen signaling (Bailey-Serres et al. 2012). Not only ethylene accumulates within plant tissues and in their surroundings underwater, but ethylene production is boosted by hypoxia as a result of the upregulation of the biosynthetic enzymes ACC synthase (ACS) and ACC oxidase (ACO) (van der Straeten et al. 2001, Rieu et al. 2005) For these reasons, ethylene plays a primary role in dictating the type of strategy that a plant would adopt to escape the flooding condition as it happens in wild-rice varieties. On the other hand, under anoxic conditions the activity of ACO is hampered, thereby reducing its impact in artificial conditions where the composition of the gaseous atmosphere is altered. Most of the ERF-VII genes (RAP2.2, RAP2.3 and HRE1) are ethylene inducible although the integration of this responsiveness to the other regulatory mechanisms activated by oxygen depletion is not deciphered yet. Mutants for ethylene signaling are impaired in their ability to endure prolonged flooding or hypoxic stress (Peng et al. 2001), although it remains to be clarified whether this is dependent on the effect of this growth regulator of ERF-VII genes or it is rather mediated via alternative pathways.

Other growth regulators do not seem to play a major regulatory role on the expression of ERF-VII genes in Arabidopsis, with the exception of HRE1 being repressed by abscisic acid and cytokinins (Goda et al. 2008, Pandey et al. 2010). The discovery of the link between germination and hypoxia through the NERP poses new interest into the effect of abscisic acid and gibberellins in the hypoxic signaling (Gibbs et al. 2011). This is further supported by the adaptive hyponastic response observed in several plant species that was also demonstrated to be orchestrated by ethylene, gibberellins and abscisic acid (Bailey-Serres and Voesenek 2008).

\section{Non-NERP ERF-VII proteins}

The control exerted by the NERP on ERF-VII depends on the cysteine residue present in their conserved $\mathrm{N}$-terminal consensus MCGGAAI (Licausi et al. 2011b). However, Cys is only one of the amino acids conserved in the sequence, suggesting that also the other five possess regulatory or structural properties. The most obvious explanation is that the composition and arrangement of this domain enables an optimal sensitivity of the Cys2 for redox changes. Under this perspective, the extremely short side-chain of the two Gly residues immediately after Cys2 might grant its exposure to the cell environment. Alternatively, this domain might provide a docking site for additional factors able to promote or reverse Cys oxidation or to anchor the TF at the plasma membrane.

Moreover, the role of this Cys-dependent branch of the NERP is unlikely to be limited to the ERF-VII factors. In plants, Methionine-Cysteine (MC) proteins represent the $0.1 \%$ of the total annotated proteome (Licausi et al. 2011b) suggesting an evolutive pressure against its occurrence. Gibbs et al. (2011) demonstrated that other MC proteins can be substrates for the NERP degradation but the presence of the Cys2 is not sufficient as determinant. Systematic mutagenesis of the conserved ERF-VII motif and of NERP-independent Cys2-containing $\mathrm{N}$-termini will be required to evaluate the whole spectrum of requirements for oxygen-dependent protein instability.

Attention should also be paid to those ERF-VII members that do not contain the conserved MCGGAI/L motif. For instance, within the Sub1 clade of rice ERF genes only Sub1b possess a complete ERF-VII consensus (Bailey-Serres et al. 2012). The N-terminus of Sub1C is composed of three Arg residues that make impossible the removal of methionine by the mitogen-activated kinase thereby causing stabilization of this TF (Gibbs et al. 2011). Sub1A represents an interesting case as it is not destabilized in vitro (Gibbs et al. 2011). Its increased stability was first ascribed to the presence of a Glu residue (instead of Ala) in the fifth position. However, restoring the MCGGAI consensus did not induce destabilization, suggesting that this TF is NERP-independent (Gibbs et al. 2011). This would also explain why overexpression of Sub1A in Arabidopsis caused developmental alterations (Peña-Castro et al. 2001) while the NERP-degraded HRE1 and HRE2 did not modify it (Licausi et al. 2010). Similarly, Snorkel1 (SK1) and SK2, the regulators of stem elongation in deepwater rice varieties, diverge in the fifth and sixth position, although their NERP-dependent stability has not been assessed yet (Hattori et al. 2009, Bailey-Serres et al. 2012). 
It is also interesting that the ERF-VII HRE1 in Arabidopsis and SIERF2 in tomato have been shown to produce alternative splicing isoforms that are devoid of the MCGGAI motif (Pirrello et al. 2006). As HRE1 is actually induced under anaerobic conditions, it is possible that an oxygen-insensitive isoform is preferentially produced, although this would remain stable after reoxygenation.

\section{NERP-independent signaling of oxygen deficiency is associated with reactive oxygen species}

Despite the core-response genes that are induced already when oxygen concentrations reach $8 \% \mathrm{O}_{2}$ in air, a vast amount of genes are specifically activated by anoxic conditions (van Dongen et al. 2009, Pucciariello et al. 2012). This cluster includes genes encoding for heat shock proteins (HSPs) and proteins generally associated with oxidative stress (ASCORBATE PEROXIDASE 2, ZAT10, ZAT12 and REVEILLE 1) (Pucciariello et al. 2012). Indeed, complete absence of oxygen but not hypoxia induces a transient accumulation of $\mathrm{H}_{2} \mathrm{O}_{2}$ in Arabidopsis seedlings that is associated with the induction of the Heat Shock Factor HSFA2 and its targets (Banti et al. 2010). The accumulation of hydrogen peroxide has been proposed to be caused by the activity of NADPH oxidases, integral plasma membrane proteins that reduce apoplastic $\mathrm{O}_{2}$ to $\mathrm{O}_{2}{ }^{-}$, which in turn can be rapidly converted into $\mathrm{H}_{2} \mathrm{O}_{2}$ (Pucciariello et al. 2012). It is possible that reactive oxygen species (ROS) production is further stimulated by the accumulation of reactive nitrogen species such as $\mathrm{NO}$, whose synthesis is stimulated by anoxic conditions (Stöhr and Stremlau 2006, Licausi and Perata 2009). ROS have also shown to modulate the induction of fermentative metabolism (Fig. 2). Baxter-Burrell et al. (2002) described a rheostatlike mechanism in which expression and activity of alcohol dehydrogenase (ADH) depends on a hypoxiaresponsive RHO-like small G protein of plant (ROP2). ROP2 appeared to be modulated by a ROP-GTPase that in turn is regulated by $\mathrm{H}_{2} \mathrm{O}_{2}$. The roles of $\mathrm{RBOH}$ activity and induction of oxidative stress-related proteins for plant survival in anoxic conditions were validated experimentally (Banti et al. 2010, Pucciariello et al. 2012). It remains to be clarified whether this set of ROSresponsive proteins is required during the exposure to anoxia or subsequently when normoxic condition are restored and therefore an oxidative burst occurs (Fig. 2).

\section{Effects of the oxygen sensing pathway on growth and development}

The identification of a direct oxygen sensing mechanisms in plants that regulates the response to anaerobiosis

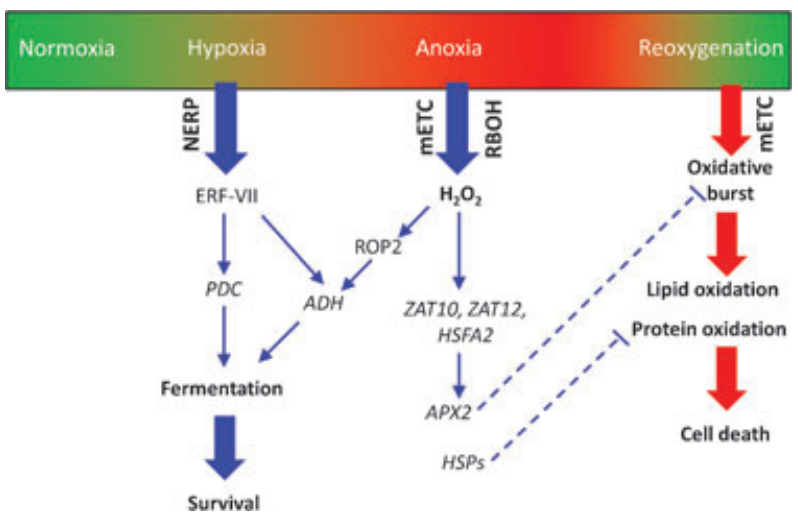

Fig. 2. ROS involvement in low oxygen signaling in Arabidopsis. Upon hypoxia, the N-end rule pathway is inhibited so that the ERF-VII TFs can induce the fermentative genes pyruvate decarboxilase (PDC) and $A D H$. When complete anoxia is reached, transient hydrogen peroxide $\left(\mathrm{H}_{2} \mathrm{O}_{2}\right)$ accumulation is promoted via the mitochondrial electron transport chain ( $\mathrm{mETC}$ ) and the plasma membrane associated NADPH oxidases (RBOH). ROS induce TFs involved in oxidative stress such as ZAT10, ZAT12 and HSFA2 that, in turn, upregulate HSPs and ascorbate peroxidase 2 (APX2). HSPs have been suggested to protect proteins and lipids from oxidative damage while APX2 acts as ROS scavenger when plants return to normoxic conditions (reoxygenation). Moreover, hydrogen peroxide enhances fermentation via ROP2. Reduction of the post-anoxic oxidative stress and enhancement of the fermentation capacity together contribute to cell survival.

suggests that the same pathway might affect growth and regulation when oxygen availability is insufficient to cope with its consumption in specific tissues or cell types. In animal systems, hypoxia directly promotes vascular development and hypoxic regions in the embryo are required to direct its spatial differentiation (Dunwoodie 2009). Similarly in maize, hypoxic cores have been demonstrated to be necessary for the establishment of the male germ line (Kelliher and Walbot 2012). In fact, hypoxic conditions $\left(1.2-1.4 \% \mathrm{O}_{2}\right)$ in young anthers provide a reductive environment for the activity of the glutaredoxin male sterile converted anther 1 (MSCA1) that triggers the germline fate in central cells (Kelliher and Walbot 2012). Artificially increasing the oxygen concentration or generating an oxidizing environment in developing anthers caused a reduction of archesporial cell number (Kelliher and Walbot 2012). Newly established germline cells secrete a diffusible peptide multiple archesporial cells 1 (MAC1) that induce the surrounding cells to differentiate into supportive tissue. This was the first report to describe the control of environment on cell fate in plants; however it is likely that oxygen concentrations play other roles in developmental processes as oxygen availability is decreased in bulks of densely packed cells. For instance, reproductive structures including fruits, 
tubers, rhizomes and seeds are likely to suffer from oxygen deficiency (Licausi et al. 2011a). Climacteric fruits stand the highest chances to develop anaerobic zones when ethylene induces the typical respiration increase. Indeed pear fruits are well known to be susceptible of browning and core breakdown upon storage in hypoxic conditions (Franck et al. 2007). Transcript analyses throughout fruit development and ripening in several plant species revealed that fermentative metabolism is induced (Chervin et al. 1999, Lara et al. 2011). In mango and melon, ripening and ethylene-induced $\mathrm{ADH}$ enzymes play a major role in the determination of fruit flavor and aroma (Manríquez et al. 2006, Singh et al. 2010). As expected, also ERF-VII genes are highly expressed in growing and ripening fruits (Lee et al. 2012). Interestingly, cross-species transcriptional comparison revealed that an ERF-VII factor is repressed during pepper berry ripening but not in tomato (Osorio et al. 2012). It is tempting to speculate that this difference might be due to a decrease in oxygen availability within the fleshy tomato berry that is instead absent in the cave pepper fruit. Also in potato tubers three hypoxia-responsive ERF genes change their expression throughout development (Licausi et al. 2011a). Moreover, artificial modification of the oxygen availability in the soil surrounding the tubers coherently altered their expression level (Licausi et al. 2011a). By means of co-expression analyses we suggested that, in these organs, ERF-VII proteins do not only regulate the anaerobic metabolism but also sucrose mobilization and starch synthesis (Licausi et al. 2011a).

\section{Conclusions}

Recent advances substantially contributed to broaden knowledge and understanding of oxygen perception and hypoxic and anoxic signaling in higher plants. From one side, the NERP for protein degradation was identified as a major component, together with a dedicated set of ERFVII TFs, in triggering the molecular response that sustains the metabolic reorganization under anaerobiosis. On the other end, ROS are beginning to appear as a key modulator of the stress response and significant factor in the determination of survival. Moreover, observations are accumulating that point out at a role for reduced oxygen conditions in the determination of cell fate and developmental or growth programs. The increase of information related to the response to hypoxia is not only essential to assist the breeding of flooding-resistant crops, but also for secondary traits that are associate with oxygen deficiency in normoxic environmental atmosphere.

Acknowledgements - I thank Pierdomenico Perata for critical reading of this manuscript.

\section{References}

Armstrong W (1979) Aeration in higher plants. Adv Bot Res 7: 225-332

Bailey-Serres J, Voesenek LACJ (2008) Flooding stress: acclimations and genetic diversity. Annu Rev Plant Biol 59: 313-339

Bailey-Serres J, Fukao T, Gibbs DJ, Holdsworth MJ, Lee SC, Licausi F, Perata P, Voesenek LACJ, van Dongen JT (2012) Making sense of low oxygen sensing. Trends Plant Sci 17: 129-138

Banti V, Mafessoni F, Loreti E, Alpi A, Perata P (2010) The heat-inducible transcription factor hsfa2 enhances anoxia tolerance in Arabidopsis. Plant Physiol 152: 1471-1483

Baxter-Burrell A, Yang Z, Springer PS, Bailey-Serres J (2002) RopGAP4-dependent Rop GTPase rheostat control of Arabidopsis oxygen deprivation tolerance. Science 296: 2026-2028

Büttner M, Singh KB (1997) Arabidopsis thaliana ethylene-responsive element binding protein (AtEBP), an ethylene-inducible, GCC box DNA-binding protein interacts with an ocs element binding protein. Proc Natl Acad Sci USA 94: 5961-5966

Chervin C, Truett JK, Speirs J (1999) Alcohol dehydrogenase expression and alcohol production during pear ripening. J Am Soc Hortic Sci 124: 71-75

van Dongen JT, Fröhlich A, Ramírez-Aguilar SJ, Schauer N, Fernie AR, Erban A, Kopka J, Clark J, Langer A, Geigenberger P (2009) Transcript and metabolite profiling of the adaptive response to mild decreases in oxygen concentration in the roots of Arabidopsis plants. Ann Bot 103: 269-280

Dordas C, Hasinoff BB, Igamberdiev AU, Manac'h N, Rivoal J, Hill RD (2003) Expression of a stress-induced hemoglobin affects $\mathrm{NO}$ levels produced by alfalfa root cultures under hypoxic stress. Plant J 35: 763-770

Dunwoodie SL (2009) The role of hypoxia in development of the mammalian embryo. Dev Cell 17: 755-773

Franck C, Lammertyn J, Ho QT, Verboven P, Verlinden B, Nicolaï BM (2007) Browning disorders in pear fruit. Postharvest Biol Technol 43: 1-13

Gibbs DJ, Lee SC, Md Isa N, Gramuglia S, Fukao T, Bassel GW, Correia CS, Corbineau F, Theodoulou FL, Bailey-Serres J, Holdsworth MJ (2011) Homeostatic response to hypoxia is regulated by the $\mathrm{N}$-end rule pathway in plants. Nature 479: 415-418

Goda H, Sasaki E, Akiyama K, Maruyama-Nakashita A, Nakabayashi K, Li W, Ogawa M, Yamauchi Y, Preston J, Aoki K, Kiba T, Takatsuto S, Fujioka S, Asami T, Nakano T, Kato H, Mizuno T, Sakakibara H, Yamaguchi S, Nambara E, Kamiya Y, Takahashi H, Hirai MY, Sakurai T, Shinozaki K, Saito K, Yoshida S, Shimada Y (2008) The AtGenExpress hormone and chemical treatment data set: experimental design, data evaluation, model data analysis and data access. Plant J 55: 526-542 
Hattori Y, Nagai K, Furukawa S, Song X-J, Kawano R, Sakakibara H, Wu J, Matsumoto T, Yoshimura A, Kitano H, Matsuoka M, Mori H, Ashikari M (2009) The ethylene response factors SNORKEL1 and SNORKEL2 allow rice to adapt to deep water. Nature 460: 1026-1030

Hinz M, Wilson IW, Yang J, Buerstenbinder K, Llewellyn D, Dennis ES, Sauter M, Dolferus R (2010) Arabidopsis RAP2.2: an ethylene response transcription factor that is important for hypoxia survival. Plant Physiol 153: 757-772

Igamberdiev AU, Hill RD (2004) Nitrate, NO and haemoglobin in plant adaptation to hypoxia: an alternative to classic fermentation pathways. J Exp Bot 55: 2473-2482

Kelliher T, Walbot V (2012) Hypoxia triggers meiotic fate acquisition in maize. Science 337: 345-348

Kwon YT, Kashina AS, Davydov IV, Hu R-G, An JY, Seo JW, Du F, Varshavsky A (2002) An essential role of $\mathrm{N}$-terminal arginylation in cardiovascular development. Science 297: 96-99

Lara MV, Budde CO, Porrini L, Borsani J, Murray R, Andreo CS, Drincovich MF (2011) Peach (Prunus persica) fruit response to anoxia: reversible ripening delay and biochemical changes. Plant Cell Physiol 52: 392-403

Lee K-W, Chen P-W, Lu C-A, Chen S, Ho T-HD, Yu S-M (2009) Coordinated responses to oxygen and sugar deficiency allow rice seedlings to tolerate flooding. Sci Signal 2: ra61

Lee JM, Joung J-G, McQuinn R, Chung M-Y, Fei Z, Tieman D, Klee H, Giovannoni J (2012) Combined transcriptome, genetic diversity and metabolite profiling in tomato fruit reveals that the ethylene response factor SIERF6 plays an important role in ripening and carotenoid accumulation. Plant J 70: 191-204

Li H-Y, Xiao S, Chye M-L (2008) Ethylene- and pathogen-inducible Arabidopsis acyl-CoA-binding protein 4 interacts with an ethylene-responsive element binding protein. J Exp Bot 59: 3997-4006

Liao Y-D, Jeng J-C, Wang C-F, Wang S-C, Chang S-T (2004) Removal of $\mathrm{N}$-terminal methionine from recombinant proteins by engineered $E$. coli methionine aminopeptidase. Protein Sci 13: 1802-1810

Licausi F (2011) Regulation of the molecular response to oxygen limitations in plants. New Phytol 190: 550-555

Licausi F, Perata P (2009) Low oxygen signaling and tolerance in plants. Elsevier Academic Press, Burlington, pp 139-198

Licausi F, Van Dongen JT, Giuntoli B, Novi G, Santaniello A, Geigenberger P, Perata P (2010) HRE1 and HRE2, two hypoxia-inducible ethylene response factors, affect anaerobic responses in Arabidopsis thaliana. Plant J 62: $302-315$

Licausi F, Giorgi FM, Schmälzlin E, Usadel B, Perata P, van Dongen JT, Geigenberger P (2011a) HRE-type genes are regulated by growth-related changes in internal oxygen concentrations during the normal development of potato (Solanum tuberosum) tubers. Plant Cell Physiol 52: 1957-1972

Licausi F, Kosmacz M, Weits DA, Giuntoli B, Giorgi FM, Voesenek LACJ, Perata P, Van Dongen JT (2011b) Oxygen sensing in plants is mediated by an $\mathrm{N}$-end rule pathway for protein destabilization. Nature 479: 419-422

Licausi F, Weits DA, Pant BD, Scheible W-R, Geigenberger P, van Dongen JT (2011c) Hypoxia responsive gene expression is mediated by various subsets of transcription factors and miRNAs that are determined by the actual oxygen availability. New Phytol 190: 442-456

Manríquez D, El-Sharkawy I, Flores F, El-Yahyaoui F, Regad F, Bouzayen M, Latché A, Pech J-C (2006) Two highly divergent alcohol dehydrogenases of melon exhibit fruit ripening-specific expression and distinct biochemical characteristics. Plant Mol Biol 61: 675-685

Mustroph A, Lee SC, Oosumi T, Zanetti ME, Yang H, Ma K, Yaghoubi-Masihi A, Fukao T, Bailey-Serres J (2010) Cross-kingdom comparison of transcriptomic adjustments to low-oxygen stress highlights conserved and plant-specific responses. Plant Physiol 152: 1484-1500

Nakano T, Suzuki K, Fujimura T, Shinshi H (2006) Genome-wide analysis of the ERF gene family in Arabidopsis and rice. Plant Physiol 140: 411-432

Osorio S, Alba R, Nikoloski Z, Kochevenko A, Fernie AR, Giovannoni JJ (2012) Integrative comparative analyses of transcript and metabolite profiles from pepper and tomato ripening and development stages uncovers species-specific patterns of network regulatory behavior. Plant Physiol 159: 1713-1729

Pandey S, Wang R-S, Wilson L, Li S, Zhao Z, Gookin TE, Assmann SM, Albert R (2010) Boolean modeling of transcriptome data reveals novel modes of heterotrimeric G-protein action. Mol Syst Biol 6: 372

Papdi C, Ábrahám E, Joseph MP, Popescu C, Koncz C, Szabados L (2008) Functional identification of Arabidopsis stress regulatory genes using the controlled cDNA overexpression system. Plant Physiol 147: 528-542

Peña-Castro JM, van Zanten M, Lee SC, Patel MR, Voesenek LAJC, Fukao T, Bailey-Serres J (2011) Expression of rice SUB1A and SUB1C transcription factors in Arabidopsis uncovers flowering inhibition as a submergence tolerance mechanism. Plant J 67: 434-446

Peng H-P, Chan C-S, Shih M-C, Yang SF (2001) Signaling events in the hypoxic induction of alcohol dehydrogenase gene in Arabidopsis. Plant Physiol 126: 742-749

Pirrello J, Jaimes-Miranda F, Sanchez-Ballesta MT, Tournier B, Khalil-Ahmad Q, Regad F, Latché A, Pech JC, Bouzayen M (2006) SI-ERF2, a tomato ethylene 
response factor involved in ethylene response and seed germination. Plant Cell Physiol 47: 1195-1205

Pucciariello C, Parlanti S, Banti V, Novi G, Perata P (2012)

Reactive oxygen species-driven transcription in Arabidopsis under oxygen deprivation. Plant Physiol 159: $184-196$

Rieu I, Cristescu SM, Harren FJM, Huibers W, Voesenek LACJ, Mariani C, Vriezen WH (2005) RP-ACS1, a flooding-induced 1-aminocyclopropane-1-carboxylate synthase gene of Rumex palustris, is involved in rhythmic ethylene production. J Exp Bot 56: $841-849$

Roberts JKM, Chang K, Webster C, Callis J, Walbot V (1989) Dependence of ethanolic fermentation, cytoplasmic ph regulation, and viability on the activity of alcohol dehydrogenase in hypoxic maize root tips. Plant Physiol 89: 1275-1278

Rocha M, Sodek L, Licausi F, Hameed M, Dornelas M, Dongen J (2010) Analysis of alanine aminotransferase in various organs of soybean (Glycine max) and in dependence of different nitrogen fertilisers during hypoxic stress. Amino Acids 39: 1043-1053

Singh RK, Sane VA, Misra A, Ali SA, Nath P (2010) Differential expression of the mango alcohol dehydrogenase gene family during ripening. Phytochemistry 71: 1485-1494

Stöhr C, Stremlau S (2006) Formation and possible roles of nitric oxide in plant roots. J Exp Bot 57: 463-470

Van der Straeten D, Zhou Z, Prinsen E, Van Onckelen HA, Van Montagu MC (2001) A comparative molecular-physiological study of submergence response in lowland and deepwater rice. Plant Physiol 125: 955-968

Xu K, Xu X, Fukao T, Canlas P, Maghirang-Rodriguez R, Heuer S, Ismail AM, Bailey-Serres J, Ronald PC, Mackill DJ (2006) Sub1A is an ethylene-response-factor-like gene that confers submergence tolerance to rice. Nature 442: 705-708

Edited by V. Hurry 